First publ. in:

Angewandte Chemie / International Edition

45 (2006), pp. 3399-3400

\title{
The Organic Chemistry of Sugars
}

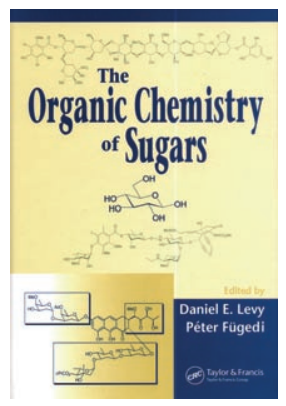

Edited by Daniel E. Levy and Péter

Fügedi. CRC Press/

Taylor \& Francis, Boca Raton 2006. 928 pp., hardcover $\$ 199.95 .-I S B N$ o-8247-5355-O

Compared with many other organic molecules, the special quality of carbohydrates, which are known to play a key role in many biological processes, is their high density of functional groups and their impact on each other. As a

Konstanzer Online-Publikations-System (KOPS)

URL: http://www.ub.uni-konstanz.de/kops/volltexte/2007/4451/

URN: http://nbn-resolving.de/urn:nbn:de:bsz:352-opus-44519 
consequence, sugars that apparently differ only slightly (e.g., in the configuration of a single center of chirality) often display a fundamentally different reactivity pattern. The transferability of reactivities, a common concept in organic chemistry, is therefore more complicated. This is probably the reason why carbohydrate chemistry is often regarded as a specialty, and is treated only superficially in textbooks and lectures on organic chemistry. The aim of this book edited by Levy and Fügedi is to oppose that perception. It consists of 4 parts with 16 chapters in total. Each chapter starts with an introduction that allows nonspecialists to also find a way of approaching the subject. Particularly successful in this respect is the first part, which has a strong textbook character. The contributions, all written all by well-known researchers, are well coordinated with each other and cover most aspects of synthetic sugar chemistry.

In the first part, comprising five chapters, basic aspects regarding structure and reactivity of carbohydrates are treated. Chapter 1 ends with a survey of the history of carbohydrate chemistry, from Emil Fischer to the beginning of the 21 st century. The second chapter is about constitution, configuration, and conformation of monosaccharides and some oligosaccharides. All the important concepts, including the anomeric and exo-anomeric effects, as well as nomenclature of oligosaccharides, are presented. Chapter 3 concerns the dayto-day business of the carbohydrate chemist, the manipulation of protecting groups. Given the fact that these operations consume a considerable amount of laboratory time, the chapter could have been a bit longer. Of course, there are many reviews on that topic, which are cited here. Nevertheless, at this point I would have preferred more examples and mechanistic explanations of the regioselectivities described. On the other hand, all the important principles are considered, and many protecting group strategies from the recent literature are mentioned. Also, the comparison of different total syntheses of trimeric Lewis ${ }^{\mathrm{x}}$ with regard to the protecting group strategy chosen is worth reading. Glycosylation methods are the subject of the following chapter, which has the highest number (640) of literature references within the book. All the relevant methods are described in a systematic way. Stereochemical aspects of glycosylations are discussed on an appropriate level. The numerous tables that allow easy access to hundreds of original publications on the use of all kinds of activators for specific types of glycosyl donors are very helpful, especially for a synthetic chemist. The first part of the book ends with a chapter on oligosaccharide synthesis. Different strategies for the assembly of these natural products, even including onepot sequential glycosylations and automated solid-phase syntheses, are described.

The second part, consisting of four chapters, covers the conversion of carbohydrates into molecules that lack some of the features that define carbohydrates. In Chapter 6 the reader is introduced to reactions for the functionalization of sugars. Besides substitutions and eliminations, the chapter discusses $\mathrm{C}-\mathrm{C}$ bond-forming reactions, redox reactions, and rearrangements. This is followed by chapters on the synthesis of C-glycosides, carbasugars, and sugars with endocyclic heteroatoms other than oxygen.

The application of sugars as tools in organic chemistry is the subject of the third part, which comprises four chapters. Chapter 10 summarizes recently published work on the use of sugars as chiral auxiliaries. That is followed by a detailed discussion of their exploitation as chiral-pool materials for the synthesis of enantiomerically pure complex target molecules. Continuing this approach, Chapter 12 concerns the syntheses of carbohydrate-containing natural products. This part ends with an overview of the latest developments (including publications up to 2005) in the area of asymmetric de novo synthesis of monosaccharides and related molecules.

In the last part, comprising three chapters, several recent developments in the glycosciences are considered. Combinatorial carbohydrate chemistry is the subject of Chapter 14. Unfortunately, recent work on carbohydrate arrays is left out. Chapter 15 deals with the preparation of glycopeptides in solution and on a solid phase, with the main emphasis on O-glycosidic structures. Unfortunately, the application of ligation reactions for the synthesis of larger glycoproteins is mentioned only briefly. The book ends with a nice overview of the use of carbohydrate mimetics as therapeutics. Using several examples, including the antiviral tamiflu, about which there has been much public discussion recently, the ups and downs on the way towards sugar-based drugs are illustrated.

The more than 3000 references that are cited could certainly have been slightly more up-to-date. In general, the literature coverage extends to 2002/ 2003. The only exception to that is the chapter on C-glycoside synthesis, which fails to cover the developments of the last ten years. In an exemplary manner, all the references include the titles in addition to the authors. This makes it easier for the reader to decide if a particular article is worth reading. The content of the book is easily accessible through the detailed table of contents as well as the index. I found only a few typographical errors. All in all, the book is highly recommended for carbohydrate chemists and those wishing to become one.

\section{Valentin Wittmann}

Fachbereich Chemie

Universität Konstanz (Germany)

DOI: 10.1002/anie.200685377 